\title{
Electric Currents Key to Magnetic Phenomena
}

\author{
Donald E. Scott*
}

University of Massachusetts/Amherst, USA

\begin{abstract}
Including the effects of electric currents in any description of the origin, shape, or motion of cosmic magnetized plasma is crucial for understanding many observed astronomical phenomena. The Maxwell (Heaviside) equations are based on real experimental measurements. These fundamental expressions clearly link electric current densities, magnetic flux densities, and electric fields into a unified conceptual whole. Examples are presented to demonstrate the pitfalls of omitting the contribution and effects of currents from descriptions of the behavior of magnetic fields. An example suggests a possible electrical explanation of the enigmatic cyclical reversal of magnetic polarities near sunspots and demonstrates the unique insight afforded by including the causal effects of currents.
\end{abstract}

Keywords: Plasma, electromagnetism, reversing solar magnetic field, solar and cosmic electric currents, magnetic reconnection.

\section{INTRODUCTION}

Ever since Gauss, Faraday, and others ${ }^{1}$ provided the experimental measurements that J. C. Maxwell and Oliver Heaviside codified into their four basic equations, there have been two different methodologies advanced in astrophysics for explaining and predicting the behavior of cosmic magnetic phenomena. One of these involves the explicit inclusion of causal electric currents and one shuns any mention of them.

The two Maxwell curl equations provide the basis of this dichotomy.

$$
\nabla \times \mathrm{B}=\mu_{0} \mathrm{~J}+\mu_{0} \varepsilon_{0} \frac{\partial \mathrm{E}}{\partial \mathrm{t}}
$$

and

$$
\nabla \times \mathrm{E}=-\frac{\partial \mathrm{B}}{\partial \mathrm{t}}
$$

where $B$ is the magnetic flux density (Webers $/ \mathrm{m}^{2}$ ), $J$ is the electric current density $\left(A m p s / \mathrm{m}^{2}\right), E$ is the electric field (V/m or Newtons/Coulomb); $\mu_{0}$ and $\varepsilon_{0}$ are, respectively, the magnetic permeability and electric permittivity of free space. All the upper case symbols in (1) and (2) represent vectors. The second term on the right in (1) is called the displacement current and is often ignored in order to simplify the equation. However it is sometimes convenient to account for the kinetic energy of magnetized plasma as being

*Address correspondence to this author at the University of Massachusetts/Amherst, USA; Tel: (480) 688-4414; E-mail: dascott3@ @cox.net. ${ }^{\#}$ Retired

${ }^{1}$ In 1821 Hans Christian Øersted in Denmark found, that an electric current caused a compass needle to move. An electric current produced a magnetic force. Andre-Marie Ampere in France soon unraveled the meaning. The fundamental nature of magnetism was not associated with magnetic poles or iron magnets, but with electric currents. The magnetic force was basically a force between electric currents.

$$
\varepsilon=\varepsilon_{0}\left[1+\left(\mathrm{c} / \mathrm{V}_{\mathrm{MH}}\right)^{2}\right]
$$

where $c$ and $V_{M H}$ are, respectively, the velocities of light and hydromagnetic waves [1]. (If this formulation is used, the displacement current is often large.) And therein lies the rub.

\section{AVOIDING CURRENTS}

Those who prefer to avoid explicit mention of electric currents solve the simplified form of (1) for the quantity $J$ (using $(\nabla \times \mathrm{B}) / \mu_{0}$ in its place). This is acceptable in describing a number of phenomena:

- Magnetic fields by themselves are measured more easily than are currents.

- Magnetic fields by themselves are basic to the study of plasma anisotropy and high-energy particle motion.

- Magnetic fields by themselves provide a good description of some waves in plasma.

However, use of magnetic fields without consideration of electric currents, cannot provide clear understanding of:

- The formation of double layers.

- Explosive events such as solar flares, and magnetic substorms.

- Formation of filaments in the solar atmosphere (corona) and the plasmasphere of Venus.

- Other phenomena that are mentioned below and are the main purpose of this paper.

Equation (2) above implicitly involves electric current in that it is the compact mathematical statement of the observation that any closed conducting loop that is linked by a timevarying magnetic field will carry an induced current in the direction that opposes the growth of that magnetic flux. (If the loop is cut, a voltage will appear across the terminals thus created). Therefore, (1) states that any current (or time 
varying electric field) will produce a magnetic field; (2) states that any time varying magnetic field that links a closed conducting path (conducting loop) will produce a voltage rise which in turn creates an electric current in the loop. Magnetic fields that obey equation (1) cannot exist in the absence of the current density, $J$, which is their cause. The only magnetic fields that do not obey equation (1) are produced by bar magnets. There are no bar magnets in space.

\section{ENIGMATIC PLASMA MOTIONS}

In descriptions that ignore the involvement of currents in plasma phenomena several questions always remain unasked (and therefore unaddressed). Basically, we must ask what causes magnetic fields to move or change strength? For example, in attempts to explain the cause of solar flares and coronal mass ejections (CMEs), 'magnetic reconnection' is usually invoked. But what is magnetic reconnection $[2,3]$.

Usually explanations of magnetic reconnection start off by saying, "Two magnetic lines of force approach each other, touch, and then separate in orthogonal directions." But the first, most basic question that must be answered is what makes those two lines move toward each other in the first place? There is no known mechanism that can grab hold of two adjacent 'lines of force' in a magnetic field and push them together. So how (why?) do they do this? Magnetic fields do not have the ability of self-initiated volition. It is impossible to answer basic questions such as this without making reference to the time varying electric currents (charge flows) that determine the shape and strength of the involved magnetic fields.

An example of an appalling lack of knowledge about how magnetic fields originate is the following extract from an article in New Scientist [4]:

Relatively confined magnetic fields like those in the Earth and Sun are generated by the turbulent mixing of conducting fluids in their cores. But large-scale fields tangled within galaxies and clusters of galaxies are harder to explain by fluid mixing alone.

Where did this writer ever get the idea that the mixing of conducting fluids creates magnetic fields? What Department of Physics teaches this?

One can take salt water (a conducting fluid) or blood (a very conductive fluid), mix it, whip it, homogenize it, boil it, pour it from great heights or even centrifuge it, or try to compress it - no magnetic fields will result from any of these actions. Magnetic fields are only created by and moved around by electric currents or other magnetic fields - but nothing else, certainly not by mixing conductive fluids.

Another example - this excerpt is taken from an article entitled Possible Origin Of Magnetic Fields In Space Uncovered [5]:

The data suggest that the ghost cavities are filled with magnetic fields, which are released into the cosmos when the cavities burst apart. This could explain the strong magnetic forces that make up the structure of galaxy clusters, according to the astronomers.

"We've known for the past 15 to 20 years that magnetic fields exist, but we didn't understand how they got there," said McNamara, an associate professor of physics and astronomy in the College of Arts and Sciences whose research is funded by NASA. "This could be a viable mechanism." [Emphasis added.]

This writer seriously suggests that the origin of magnetic fields is "ghost cavities that burst apart in the cosmos."

One more example (hundreds of such are easily obtainable via an Internet search on the topic: What causes magnetic fields?): in 2008 a NASA report contained the following [6]:

NASA's five THEMIS spacecraft have discovered a breach in Earth's magnetic field ten times larger than anything previously thought to exist.

The magnetosphere is a bubble of magnetism that surrounds Earth and protects us from solar wind. The event began with little warning when a gentle gust of solar wind delivered a bundle of magnetic fields from the Sun to Earth. Like an octopus wrapping its tentacles around a big clam, solar magnetic fields draped themselves around the magnetosphere and cracked it open. [Emphasis added.]

Magnetic fields are not delivered in bundles. And the solar wind does not move them around. Magnetic fields are created by and moved around by electric currents - nothing else.

\section{MAGNETICALLY STORED ENERGY}

FARADAY'S LAW - The formal statement of Faraday's Law is, "The induced electromotive force or EMF in any closed circuit is equal to the time rate of change of the magnetic flux linking the circuit." This is a restatement of expression (2) above. In the case of a coil of wire wrapped around a material core carrying magnetic flux, $B$, the voltage across the terminals of the coil is given by

$$
\mathrm{v}=\frac{\mathrm{dn} \phi}{\mathrm{dt}}
$$

where $\phi$ is the total magnetic flux in Webers (which is $B$, the flux density in Webers per square meter integrated over the cross-section of the coil). But in any electrical element that has voltage, $v$, across it and current, $i$, through it, the energy stored in that element is

$\mathrm{W}\left(\mathrm{t}_{0}\right)=\int_{-\infty}^{\mathrm{t}_{0}} \mathrm{v}(\mathrm{t}) \mathrm{i}(\mathrm{t}) \mathrm{dt}$

thus, for a constant number of turns, $n$, we have

$$
\begin{aligned}
& \mathrm{W}\left(\mathrm{t}_{0}\right)=\int_{\mathrm{t}=-\infty}^{\mathrm{t}=\mathrm{t}_{0}} \frac{\mathrm{d}(\mathrm{n} \phi)}{\mathrm{dt}} \mathrm{i}(\mathrm{t}) \mathrm{dt} \\
& =\int_{\phi(-\infty)}^{\phi\left(\mathrm{t}_{0}\right)} \text { ni } \mathrm{d} \phi
\end{aligned}
$$

If the relationship between the magnetic flux $\phi$ and the applied ampere-turns, $n i$, is linear

$\phi(\mathrm{t})=\mathrm{k}$ ni

then (7) becomes 


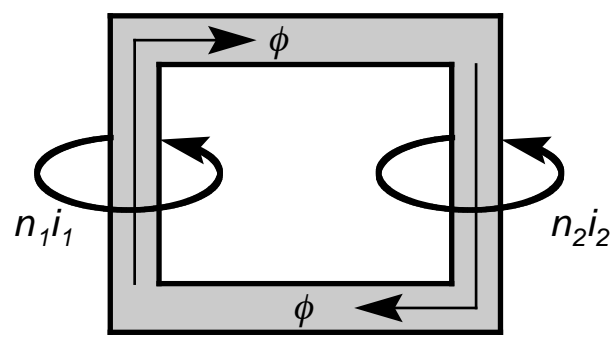

Fig. (1). A simple magnetic circuit.

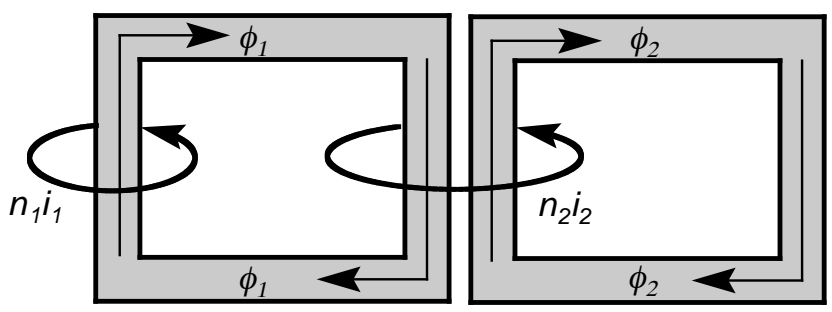

Fig. (2). Two magnetic paths linked by an induced secondary current.

$\mathrm{W}\left(\mathrm{t}_{0}\right)=\int_{\phi(-\infty)}^{\phi\left(\mathrm{t}_{0}\right)}$ nikn di

or $\mathrm{W}\left(\mathrm{t}_{0}\right)=\frac{\mathrm{kn}^{2}}{2} \mathrm{i}\left(\mathrm{t}_{0}\right)^{2}=1 /(2 \mathrm{k}) \phi\left(\mathrm{t}_{0}\right)^{2}$

The energy stored in the magnetic field structure at any instant, $t_{0}$, is proportional to the square of the current, $i\left(t_{0}\right)$. Similarly, the total energy stored in the magnetic field at any time, $t_{0}$, is proportional to the square of the total magnetic flux at that instant, $\phi\left(t_{0}\right)$. If the current is reduced to zero value, all the energy previously stored in the field is released.

\section{MAGNETIC CIRCUITS}

Equation (8) suggests that application of the forcing function, $n i$, to a magnetically linear material (free space, air) results in the production of a magnetic flux, $\phi$, in much the same way that the application of a voltage, $v$, across a resistor produces a flow of charge, $i$.

For simplicity in the example sketched in Fig. (1), both $n_{1}$ and $n_{2}$ are set to unity. The input current, $i_{l}$, produces flux, $\phi$. The linear relationship between those quantities implies that if $i_{l}$ increases, then $\phi$ will increase at an equal rate.

If $i_{l}$ is held constant (is time-invariant), then $\phi$ will also be time-invariant, and there will be no current induced in the second winding (so $i_{2}$ will be zero valued). Equations (2) and (4) state that only a time varying magnetic field can induce a non-zero valued $i_{2}$. What (2) and (4) actually say is that, if we cut the conducting loop, $n_{2}$, creating two terminals, a voltage, $v_{2}$ will be measured across those terminals that would force current $i_{2}$ to flow in a resistor connected to those terminals. The minus sign in equation (2) indicates that the induced (secondary) current will be in a direction that tends to oppose the growth of the magnetic flux. So the strength of $i_{2}$ is determined by the time rate of growth of $\phi$.

Consider a slight extension to the first example. See Fig. (2). Suppose the input current, $i_{l}$ is now a time varying signal
- one that never reverses direction, but can get stronger and weaker.

As a result of the variation in $i_{l}$, the magnetic flux, $\phi$, will also strengthen and weaken accordingly. If $i_{l}$ does not reverse its direction, neither will $\phi_{1}$. But, because of its strengthening and weakening, the time rate of flux growth, $\partial \phi_{1} / \partial t$, will alternate in sign. Therefore, $i_{2}$, which at every instant flows in a direction to oppose the growth of $\phi_{l}$, will also reverse its direction. This causes $\phi_{2}$ to reverse its direction.

The conclusion that can be drawn from these examples is that a unidirectional current, $i_{1}$, if it varies in strength over time, can produce a magnetic flux, $\phi_{2}$, that reverses direction. This effect is utilized in the electric power industry in transformers and so is called transformer action.

\section{MAGNETIC FIELDS THAT REVERSE THEIR POLARITY}

Are there any examples of astronomical magnetic fields that occasionally reverse their direction? And, if so, do we know what causes them to do that?

Eugene N. Parker [7] correctly calls coronal loops 'bulges' in the Sun's magnetic field. He states: "The bulges emerge through the surface of the Sun, forming bipolar magnetic regions, or magnetically active regions, with lengths up to $200,000 \mathrm{~km}$. The bipolar fields have opposite signs on opposite sides of the equator, and the algebraic signs of the fields reverse from one 11-year [sunspot] cycle to the next." [Emphasis added]

This remains, for Parker, an enigmatic observation. Perhaps if he were more amenable to consideration of an electric current causality, a clearer understanding might dawn. In light of the previous example, we offer a possible explanatory mechanism in Fig. (3), below.

According to Alfvén's stellar circuit [1], an electric current (charge flow) enters or leaves each pole of the Sun. 


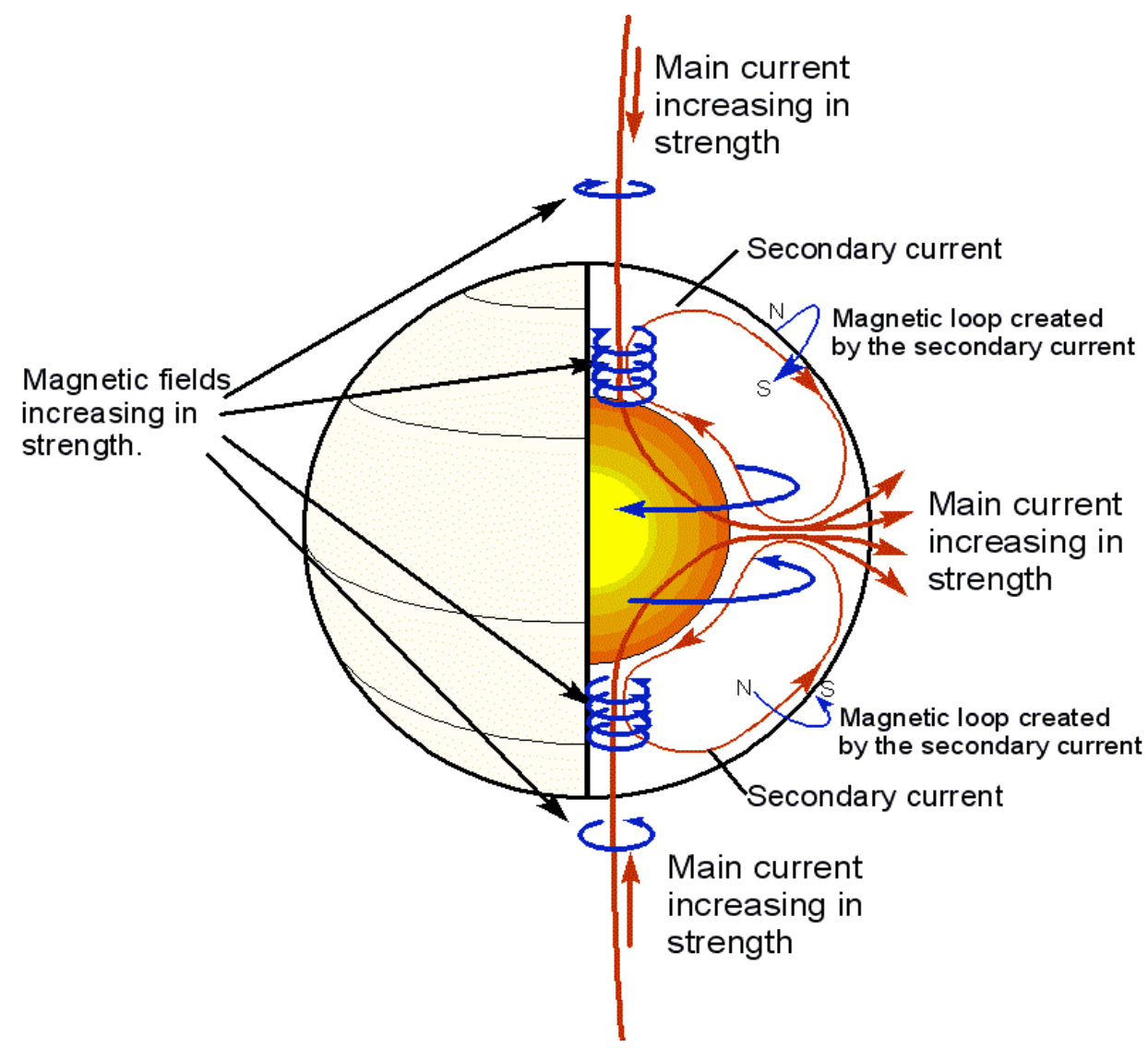

Fig. (3). Primary and secondary electric currents in the Sun.

Making use of the right-hand rule we can visualize the directions of the encircling magnetic field created by that current. If the strength of this current is increasing, the magnetic field will strengthen as well. Such time varying magnetic fields can induce secondary currents ${ }^{2}$ as shown in the earlier examples and also in Fig. (3). The secondary current will only exist (have non-zero value) when the magnitude of the primary magnetic field is growing or shrinking.

If a secondary current filament is flowing southward from near the Sun's north pole and it is on or just beneath the Sun's surface, a looping magnetic field will emerge to the east of the current creating a north magnetic pole there. (Right thumb directed toward the south, fingers emerging up out of the surface on its east side.) The loop will move out above the Sun's surface and then return down into the surface forming a south magnetic pole to the west of the current. Recall that a 'north magnetic pole' is a region where the magnetic flux emerges from a solid. ${ }^{3}$ In the Sun's southern hemisphere, the secondary surface current is flowing northward toward the solar equator. The resulting magnetic field will emerge (north magnetic pole) to the west of the current and return down to the surface (forming a south magnetic pole) to the east of the current.

\footnotetext{
${ }^{2}$ A secondary current will always flow in a direction that tends to oppose the growth of the magnetic field that induces it. This relationship can be seen in figures 2 and 3 .

${ }^{3}$ The end of a compass needle marked " $\mathrm{N}$ " is indeed a north magnetic pole. It points almost toward Earth's north pole. Thus, the region near Earth's North Pole (toward which the compass points) is, in reality, a south magnetic pole. A magnetic field is leaving the compass needle and flowing into the earth near the north geographic pole. That field then comes out of the Earth near the geographic south pole (creating there a north magnetic pole) and then flows into the end of the compass needle marked " $S$ ".
}

Regardless of the direction of the main driving current coming into the Sun, the eleven-year reversal of the magnetic loops can be explained by transformer action as shown above. If the main magnetic field that induces the surface currents is growing in strength, the surface current will point in one direction. If the main magnetic field starts to weaken in intensity, the secondary (surface) current will reverse direction. Consequently the magnetic polarity of the Omega loops will also reverse. Notice that this mechanism does not require the main solar driving current itself to reverse direction, only to vary in amplitude. Thus the action described by Parker ("The bipolar fields have opposite signs on opposite sides of the equator.") follows directly from Alfvén's circuit.

The presence of sub-surface electric currents on the Sun is not just mere speculation. In August 1997, scientists at Stanford University [8] announced that, using the joint European Space Agency (ESA)/NASA Solar and Heliospheric Observatory (SOHO) spacecraft, they had discovered 'jet streams' or what they called 'rivers of hot, electrically charged gas' (plasma) flowing beneath the surface of the Sun. They also found features similar to trade winds that transport this 'gas' below the Sun's surface. Flows of electric charges such as these are, by definition, electric currents. The image [9] that accompanied the press release has an almost one-to-one correspondence with Fig. (3).

So these reversing magnetic fields on the Sun's surface provide an archetypical example of an observed phenomenon that cannot be understood without reference to the electric currents that cause it. And even though data supporting the electrical model proposed here were published as long ago as 
1997, mainstream astronomers have not yet begun to acknowledge the importance of electric currents - neither on the Sun, or anywhere else for that matter.

\section{CONCLUSION}

The well-known inter-relationships between electric currents and magnetic fields so succinctly described a century ago by Maxwell, together with the analytical tools of modern circuit analysis, now offer investigators in astrophysics an expanded set of techniques and concepts by which they can advance their understanding of what otherwise will remain, for them, 'enigmatic' observations. The obstinate refusal of astrophysicists to acknowledge the efficacy of electric currents in the cosmos is a self-imposed obstacle to their future progress. They would do well to remove it.

\section{ACKNOWLEDGMENT}

None Declared.

\section{CONFLICT OF INTEREST}

None Declared.

\section{REFERENCES}

[1] Alfvén H. Double layers and circuits in astrophysics. IEEE Trans Plasma Sci 1986; vol. PS-14: pp. 779-793

[2] Scott DE. Special issue on space and cosmic plasma. IEEE Trans Plasma Sci 2007; 35(4). http://members.cox.net/dascott3/IEEETransPlasmaSci-Scott-aug2007.pdf

[3] Scott DE. The Electric Sky. Portland: Mikamar Publishing 2006.

[4] http://www.newscientist.com/article/dn8544-how-the-universesfirst-magnetic-field-formed.html

[5] http://www.unisci.com/stories/20021/0109021.htm

[6] http://science.nasa.gov/science-news/science-atnasa/2008/16dec_giantbreach

[7] Parker EN. The physics of the sun and the gateway to the stars. Phys Today 2000; 53: 26-31.

[8] http://soi.stanford.edu/press/ssu8-97/ssu.html

[9] http://solar-center.stanford.edu/images/plasmacom.jpg

Received: April 21, 2011

Revised: May 19, 2011

Accepted: May 19, 2011

(C) Donald E. Scott; Licensee Bentham Open.

This is an open access article licensed under the terms of the Creative Commons Attribution Non-Commercial License (http://creativecommons.org/licenses/by-nc/3.0/) which permits unrestricted, non-commercial use, distribution and reproduction in any medium, provided the work is properly cited. 